\title{
Towards Designing Health Monitoring Interfaces for Low Socioeconomic Status Families
}

\author{
Danish U. Khan, Katie A. Siek, and Swamy Ananthanarayan \\ Department of Computer Science, University of Colorado Boulder \\ Email: $\{$ khand, ksiek, ananthas $\} @$ colorado.edu
}

\begin{abstract}
We conducted a study with eight low socioeconomic status caregivers, the "gatekeepers" to family and community health, to explore their technology perceptions and visualizations that can assist them in managing their families' health. We studied how to visualize the everyday health habits of a population that is at risk for chronic illness. Through semi-structured interviews and prototype design activities, we found that the caregivers wanted to use mobile technology to monitor a subset of their family members for at least one health metric. We inform future work with an analysis of the target population's needs in relation to longitudinal health visualizations with multiple family member data streams.
\end{abstract}

\section{INTRODUCTION}

Current health monitoring technologies can provide individuals with the ability to capture detailed information about their everyday life and reflect on this data to improve their health or monitor chronic conditions. This data could be saved in a personal health management application, such as a Personal Health Record (PHR) repository, to create a lifelong record for an individual. In turn, individuals could share their information with health providers to receive personalized health recommendations. Indeed, mid to high socioeconomic status (SES) populations can currently take part in this process by utilizing smart phone applications, PHRs, and continued partnerships between PHR vendors and healthcare institutions.

Although we can collect and store a lifetime of health information, researchers have not identified how to visualize the plethora of data. Imagine if a person could easily track their food consumption, physical activity, and mood for their entire life. In this case, individuals must be able to look at their personal health data through specific time periods in varying abstractions of detail to understand how their everyday actions affect their health. In addition, since caregivers are recognized as the "gatekeepers" to family and community health, we must empower caregivers to assess their dependent family members' health to make informed health decisions.

As a first step in our user-centered design process to support life-long health information visualization, we sought to identify how individual and family-based personal health information should be visualized for low SES caregivers to make informed decision about their own and family members' health. We worked with low SES caregivers because they and their families could benefit the most from low-cost health management systems such as PHRs, given their predisposition for chronic illnesses and limited access to healthcare [1]. To this end, we conducted semi-structured interviews and prototype design activity sessions with eight caregivers from different families to understand what, why, and how caregivers would like to track and visualize their health information. In this study, we present the qualitative findings and artifacts that provide a glimpse into the design of personal health management applications. Specifically, we explored information flow, health data granularity in relation to time, and multi-data streams that represent people.

We found that caregivers preferred horizontal information flows to look at specific health metrics, but wanted a vertical information flow to look at the granularity of health data. Overall, the caregivers' preferences on data granularity varied depending on whether they wanted to input or view data, but daily granularity would meet all of their needs. Caregivers typically wanted to view a subset of their family members' data streams. The specific contributions of this note are:

- Identification of the health metrics low SES caregivers want to track for themselves and their family members.

- Designs for single person and multiple person data stream visualizations for life-long health information.

\section{Methodology}

We collaborated with the Bridge Project, a community outreach project that serves over 500 ethnically diverse, low SES families in Denver public housing communities. The population is considered low SES because $73 \%$ of children in this community receive free/reduced lunch and $41 \%$ of families live under 2 orders of magnitude of the federal poverty line. We chose this population because researchers showed that $87 \%$ of children in the Bridge Project neighborhoods were at risk for cardiovascular disease [1] and thus could benefit from self-management applications.

After we received human ethics approval, we worked with the Bridge Project personnel to recruit caregivers who lived in the community, spoke English, and had at least one child under eight years old. Teenagers played a significant role in caring for siblings and preparing meals, thus we expanded the scope of the project to include parent and teenage caregivers.

\section{A. Meetings}

Since the target population was new to health management applications, we paired our study with a simple health monitoring activity where participants took pictures of things that reminded them of health for four weeks [2]. This activity made 


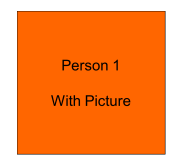

Nutrition Information (or whatever the person is monitoring) Option for grouping together stuff. E.g, Nutrition, Exercise.
Groceries, Weight, etc)

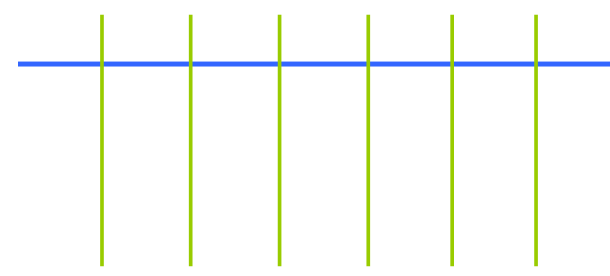

(a) Vertical Information Flow

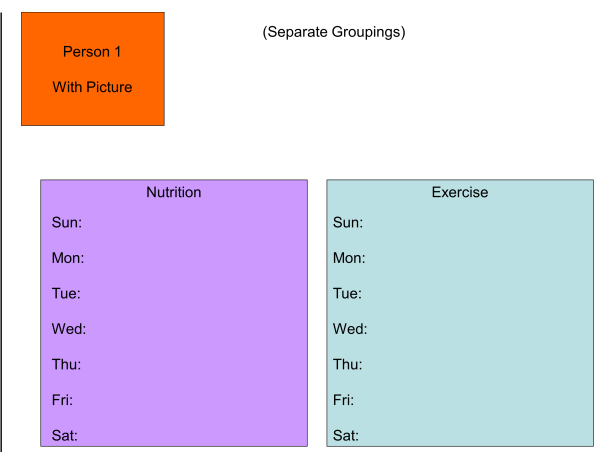

(b) Multiple Data Table

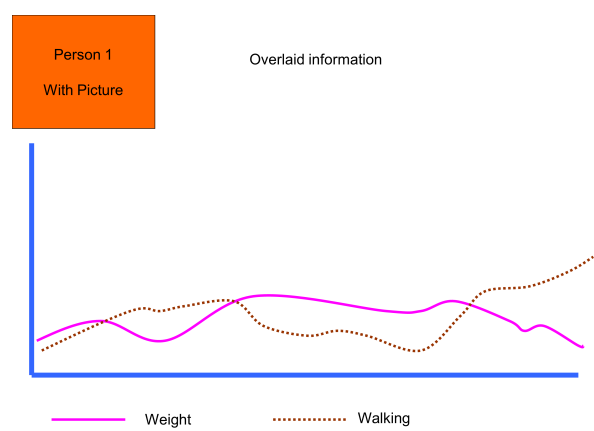

(c) Overlaid Line Chart

Fig. 1. A subset of meeting 2 prototypes for visualizing a single health data stream. Not shown - horizontal version of (a) and calendar visualization.

participants more aware of the interactions necessary to capture health data for later visualization and reflection. During the study, we met with participants individually three times to complete design exercises and semi-structured interviews. Participants received a $\$ 10$ gift card incentive per meeting.

During the first meeting, we conducted semi-structured interviews to explore what health metrics participants want to monitor and why. We then investigated which technological platforms would be most beneficial. Finally, we asked the participants how often and long they could foresee themselves using a potential health monitoring application.

In the second meeting, we focused on visualizing a single health data stream - the health data generated by one person - through prototyping activities. We presented them with five prototype interfaces - a vertical information flow (Figure 1 1a), horizontal information flow (similar to Figure 11a, but horizontal), multiple data table (Figure 1p), overlaid line chart (Figure 1F), and calendar. We showed the prototypes on a touchscreen tablet that visualized health data of a relatable persona based on their preferred trackable health metric to obtain their preferences. We used a touchscreen tablet because it provided an easy way to capture digital artifacts. Finally, participants drew a prototype on the tablet that visualized their preferred health metric.

The third meeting also consisted of similar prototyping activities where five prototype interfaces were discussed that focused on visualizing an individual's family health and therefore displayed multiple health data streams. The tabular top-bottom orientation prototype (Figure $2 \mathrm{a}$ ) visualized health information of different family members in tables. A sideby-side orientation was also used. The longitudinal weight prototype (Figure 2 2 ) was designed to track and compare two individuals' weight. We also used a prototype similar to Figure 1k, where the weight of two individuals was overlaid for comparison. Finally, the weekly comparison (Figure 2k) showed how different family members faired during a specified period of time. Afterwards, participants drew a prototype on the tablet that visualized their preferred family's health metrics.

\section{B. Analysis}

We analyzed transcriptions and participant sketched artifacts by systematically coding these with NVivo 9 . We utilized both, a deductive and inductive approach that allowed new themes to emerge from the data.

\section{Participants}

We recruited eight participants in total - four mothers and four teens from different families. The average age of the mothers was 32.8 years (s.d. $=5.4$ years). Mothers were unemployed and single, and had an average of 3.5 children. All of the mothers had access to a computer, but only two had a computer at home. All mothers owned a mobile phone and were proficient in using their mobile phones. The average age of the four female teenagers was 16.3 years (s.d. $=1$ year). On average, they had 6.5 siblings they helped care for. All the teenagers had access to a computer and all, except one, regularly used it. Three teenagers had a mobile phone and comfortably used the basic mobile phone features.

\section{RESUlTS AND DISCUSSION}

In this paper, we focus on the tractable issues of personal health and wellness management with low SES caregivers. We found that the low SES caregivers were interested in monitoring their health with a mobile phone application that they could use daily for 5 to 10 minutes. The caregivers wanted to monitor their diet daily. Participants preferred an overlaid information interface (Figure 11) for quantifiable health metrics about one person (single data stream). Most of the participants thought that the multiple health data stream visualizations would be useful for tracking their family's health. The prototype interfaces drawn by the participants were heavily influenced by the prototype interfaces shown to them.

\section{A. Preferred Health Metrics to Monitor}

Mothers were interested in tracking their family's unhealthy food consumption. Although diet was an important health metric, the mothers lacked nutritional knowledge (e.g., carbohydrates). $\mathrm{M} 2^{1}$ said, "Because I don't know too much about

${ }^{1}$ Mothers are referred to as M[Participant \#] and Teenagers are referred to as T[Participant \#]. 


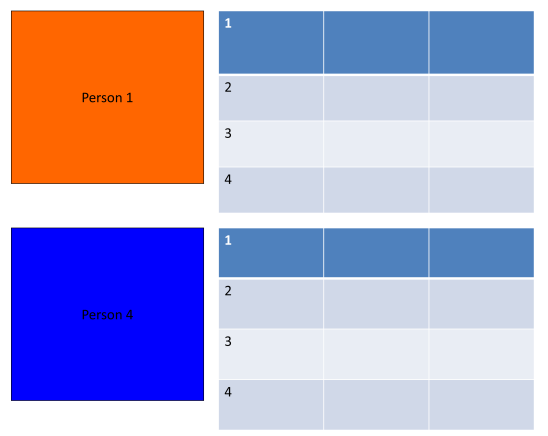

(a) Tabular Top-Bottom Orientation

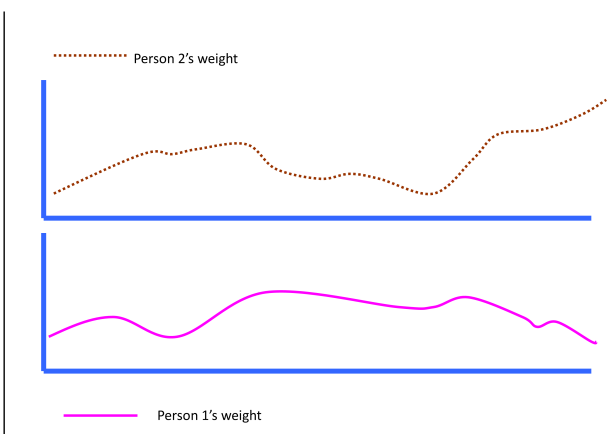

(b) Longitudinal Weight

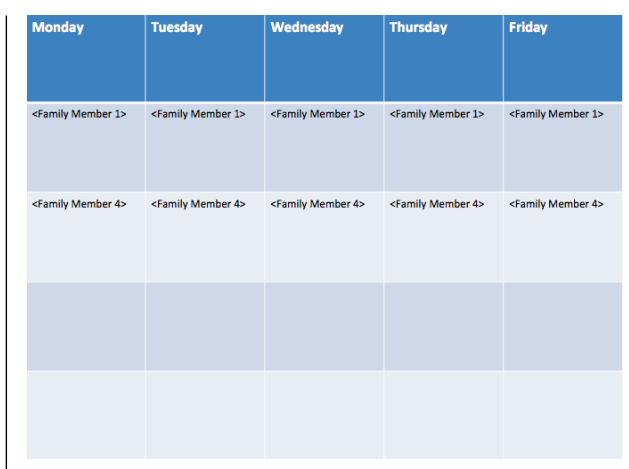

(c) Weekly Comparison

Fig. 2. A subset of meeting 3 prototypes for visualizing multiple health data streams. Not shown - side-by-side version of (a) and overlaid visualization.

carbohydrates. If I had more knowledge on what carbohydrates and all that was [pause] I would be like he ate this many carbs." Other health metrics included fluid intake and weight. With tracking weight, we observed a difference of opinion - while some caregivers found it useful, others were uncomfortable discussing it. M3 said, “...Unless you want typical people that will be like wooo so worried about the weight. It's just the weight type. Some people do worry about their weight and some just can't picture their weight or some just can't [pause] you know [pause] weigh themselves [pause] I think weight you shouldn't have in it. A lot of children get uncomfortable about their weight ..." Teenagers were interested in monitoring the effect of exercise on their weight.

While most mothers were interested in managing their children's dietary health, most of the teenage caregivers were more interested in monitoring themselves and their siblings' health. One mother was specifically interested in finding balance in her children's diet and activity levels, M2 said: "When they are active and busy and doing stuff, they hardly want to eat too versus when they just want to lie on ground and watch TV and not do anything they will eat more. So I gotta find a way to not having them too over excited to where they don't wanna eat and too lazy that they want to eat too much. So I want to find the balance." Whereas for teenagers, monitoring their parents' health was considered "not cool" and interestingly, they wanted to monitor their active siblings' health, not their inactive siblings who they considered "couch potatoes".

Although prior studies have confirmed that individuals wanted to monitor their own health [3], the literature is divided on the issue of sharing health information with family [3, 4]. While we know that the caregivers from the target population were interested in monitoring their family's health, more work is required to explore the subtle privacy concerns related to sharing various health metrics within family members.

\section{B. Technological Platforms}

After using the simple health monitoring application, two mothers and three teens preferred a mobile phone to monitor their health, whereas the remaining two mothers and one teenager preferred a computer because they said they had it at their home and used it regularly. Although all participants had cable television, participants thought that the lack of mobility made the television a secondary choice despite digital cable providing a two-way communication medium. In addition, M1 mentioned, "Even if it is on TV, there is always something else better than that to watch." Participants thought they would use the technology once a day in the afternoon, but not necessarily every day. Five participants were willing to use the application for 10 or more minutes, whereas three participants would use it for less than 10 minutes a day.

\section{Granularity of Capturing and Viewing Health Data}

Mothers and teens wanted to capture health data daily. Mothers wanted to view data on a weekly and tri-monthly granularity, whereas teens wanted to view their data daily or weekly. Although mothers said that they would ideally capture their health data on a daily basis, they were skeptical about doing so given their busy schedules. M1 said "People like me single parent [pause] I am kind of busy. And everyday I might forget one or two days. So I would do the week one."

\section{Visualizing Single Health Data Stream}

A key factor in visualizing health metrics was information orientation. For the table-based interfaces, the participants preferred a horizontal information flow for tracking health metrics, while they preferred a vertical information flow for representing time. After discussing single health metric prototypes, we showed the participants multiple data tables visualizations (Figure 1 $1 \mathrm{p}$ ). Most of the participants found it useful, however two mothers mentioned that there was "too much going on" in these prototypes. Caregivers found that an overlaid line chart (Figure 1k) was intuitive when the health metrics were related (e.g., weight and walking) and quantifiable. For unquantifiable metrics (e.g., foods consumed and exercise), they preferred a table comparison of the two metrics separately. They did not find the calendar prototype useful because of crowded information. Based on our findings, we encourage consumer health technology designers to use different visualizations based on whether the health metrics are quantifiable and related. While we designed and evaluated traditional graph and table based interfaces, we need to explore other visualization avenues such as serial periodic data 


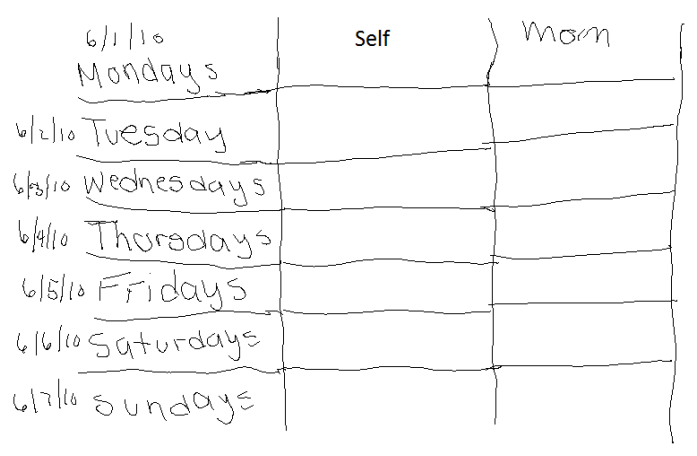

Fig. 3. T3's anonymized sketched artifact

visualizations [5] where time continues to flow serially and days and weeks occur as periodic blocks. Moreover, we also need to identify short-term health data visualization needs and compare them with longitudinal representations as research has shown that individuals find different values in both types of visualizations [6].

\section{E. Visualizing Multiple Health Data Streams}

Overall, the caregivers found value in multiple data stream visualizations because they were able to compare their family members' health metrics. Caregivers were divided over their preference for a side-by-side or top-bottom comparison (Figure 2a) of health data streams. Similarly, the participants were divided over whether multiple individuals' health information should be overlaid in a line graph. Participants preferred textual information instead of pictorial or graph representations. M2 said “... may be occasional picture here and there,", but overall the participants preferred textual information. Our findings showed that multiple health data stream interfaces should be flexible so that participants can customize the data streams' visualization orientation according to their preferences. Although other researchers have used graph-based interfaces to visualize multiple health data streams from a family perspective [7], more work is required to identify how can we best represent multiple individuals' health data for useful health information comparison.

\section{F. Prototype Interfaces Designed by the Caregivers}

When participants designed their own interfaces to visualize their family's health information, we found that a majority of the participants drew tabular interfaces. Figure 3 shows a representative drawing from $\mathrm{T} 3$ who compared her health with her mom's health. T3 also detailed her desired data granularity by providing all the days of a week. An interesting problem arose during the prototyping activities was how to visualize aggregation of food data. Although most of the participants found it difficult to visualize, T2 suggested to distinguish different foods by a color code. For example green could represent vegetable intake, while red could represent meat intake. These colored foods could be shown in a stacked bar chart to represent intake at a daily to monthly granularity.
Aside from designing traditional table-based and graphbased interfaces [8], we must explore interfaces where health information is understood in a single glance. The informatics community has designed glanceable displays for smart phones [9, 10], however to assist the target population, we must explore how glanceable displays could be designed for low-end mobile phones.

\section{G. Limitations}

We acknowledge that we had a small number of participants (8 caregivers), however there have been HCI oriented studies with a similar number of subjects [11, 12]. We also found that during the prototyping activities, most of the participants drew prototypes that were informed by our prototype designs. Despite these limitations, we assert that this preliminary study provides insights into designing family-based health monitoring visualizations.

\section{CONClusion And Future Work}

We explored how low SES caregivers would prefer to visualize specific health metrics for family members and found that caregivers would like to monitor at least one health metric for a subset of family members on a mobile device. In addition, caregivers preferred overlaid graphs to easily decipher quantifiable health metrics for family members. We aim to design and evaluate more high fidelity prototype designs to evaluate with this population. The preliminary work here provides insights into the design of future health applications and time-related data.

\section{ACKNOWLEDGMENTS}

We thank the Bridge Project based in Denver's public housing neighborhoods. Funding for this research was provided by National Science Foundation Award No IIS-0846024.

\section{REFERENCES}

[1] A. J. Barton, L. Gilbert et al., "Cardiovascular risk in hispanic and nonhispanic preschoolers." Nurs Res, vol. 55, no. 3, pp. 172-179, 2006.

[2] D. U. Khan, S. Ananthanarayan, and K. A. Siek, "Exploring everyday health routines of a low socioeconomic population through multimedia elicitations," J Participat Med., vol. 3, no. e39, 2011.

[3] J. S. Beaudin, S. S. Intille, and M. E. Morris, "To track or not to track: user reactions to concepts in longitudinal health monitoring." JMIR, vol. 8, no. 4, 2006.

[4] A. Grimes, D. Tan, and D. Morris, "Toward technologies that support family reflections on health," in GROUP, 2009, pp. 311-320.

[5] J. V. Carlis and J. A. Konstan, "Interactive visualization of serial periodic data," in UIST, 1998, pp. 29-38.

[6] M. Lee and A. Dey, "Embedded assessment of aging adults: A concept validation with stakeholders," in PervasiveHealth, 2010, pp. 1-8.

[7] N. Colineau and C. Paris, "Motivating reflection about health within the family: the use of goal setting and tailored feedback," UMUAI, pp. 1-36, 2010 .

[8] A. Ahtinen, S. Ramiah et al., "Design of mobile wellness applications: identifying cross-cultural factors," in OZCHI, 2008.

[9] S. Consolvo, D. W. McDonald et al., "Activity sensing in the wild: a field trial of ubifit garden," in CHI, 2008, pp. 1797-1806.

[10] J. Pollak, G. Gay et al., "It's time to eat! using mobile games to promote healthy eating," Pervasive Computing, vol. 9, no. 3, pp. 21 -27, 2010.

[11] K. A. Siek, K. H. Connelly, and Y. Rogers, "Pride and prejudice: learning how chronically ill people think about food," in CHI, 2006, pp. 947-950.

[12] B. K. Smith, J. Frost et al., "Facilitating narrative medical discussions of type 1 diabetes with computer visualizations and photography." Patient education and counseling, vol. 64, no. 1-3, pp. 313-321, 2006. 